\title{
P300 in Schizophrenia: Interactions between Amplitudes and Topography
}

\author{
Werner Konrad Strik, Thomas Dierks, Ernst Franzek, Gerald Stöber, and \\ Konrad Maurer
}

\begin{abstract}
Low P300 amplitudes and topographical asymmetries have been reported in schizophrenic patients, but reference-independent amplitude assessment failed to replicate reduced amplitudes. P300 amplitude is conventially assessed at midline electrodes (Pz), and asymmetric topography as reported in schizophrenics, may confound this measurement. We investigated the possible interaction berween $P 300$ ropography and assessments of amplitudes. In 41 clinically stable schizophrenics and 31 normal controls, the general finding of reduced amplitudes at the Pz-electrode and topographical asymmetries in the patient group were replicated. In both groups, asymmetries of the P300 field (lateralized peaks) reduced the standard amplitude assessment at the midline parietal electrode, but did not affect the reference-independent, global amplitude assessment. This shows that asymmetry per se does not imply reduced field strength. In addition, in schizophrenics, but not in controls, there was a significant effect of the direction of asymmetry on both amplitude measures, amplitudes being lower with increasing shift of the $P 300$ peak to the right side. Considering also the slightly left-lateralized peaks in the normal controls, this suggests that only right-lateralized $P 300$ peaks express functional deficirs in schizophrenics, whereas left-lateralized peaks fall within the physiological variability of the P300 field. The reference-independent amplitude assessment is proposed for unambiguous amplitude assessment in order to better define the clinical, psychological and physiopathological meaning of the P300 alterations in schizophrenics.
\end{abstract}

Key Words: Event-related potentials, P300, P300 topography, Brain mapping, Schizophrenia

\section{Introduction}

Low P300 amplitudes have repeatedly been reported in schizophrenia (Roth et al 1972; Pritchand 1986); however,

Address reprint requests to Wemer K. Strik, MD. Laborntory of Clin. Neurophysioloty. Dept of Psychiatry. University Hospital, Fictskeinstr. 1S, D-97000 Wurz burg, Cermany.

Department of Psychistry, University Hospital, Wurzburg/Germany: Department of Psychiatry, University Hospital, FrankfurtiGermany (WKS, TD KM)

From the Lboratory of Clinical Neurophysiology, supported in part by gants of the Deutsche Forschungsgemeinschuff (DFG).

Received February 9, 1993, revised December 7, 1993. there are indications of state-dependent variability (Duncan et al 1987) and of relevant group effects, as residual schizophrenic patients have significantly lower $\mathrm{P} 300$ amplitudes than fully remitted patients (i.e., cycloid psychoses; Strik et al 1933a). On the other hand, multichannel recordings have revealed right hemispheric lateralizations of the $\mathrm{P300}$ positivity (Morstyn et al 1983; Strik et al 1993a) and, accordingly, reduced left hemispheric activity (Faux et al 1990, see also McCarley et al 1991). Both alterations, left smaller than right hemispheric asymmetries, and low amplitudes were found to be correlated with volume reduction of the left 
posterior temporal gyrus in schizophrenics (McCarley et al 1993).

P300 is supposed to result from the activity of at least two different generators localized in the left and right medial temporal lobe; and probably other cerebral structures such as the frontal lobe (Wood and McCarthy 1985), amygdala (Halgren et al 1986), and the inferior parietal lobe (Smith et al 1990) are also involved. Normal P300 fields usually show a midline central or parietal peak, and this supposedly is the sum of the widespread generator activities. No neuroanatomical rationale (i.e., vicinity to the supposed generator) is therefore available for the convention of selecting a midline electrode (e.g. Pz) as the active electrode, but rather the fact that the peak of a typically distributed P300 is located in this region.

If there are asymmetries of the generator activity, however, topographical deviations of the $\mathrm{P} 300$ peak from midline must be expected. The overall P300 field strength, on the other hand, is not necessarily affected by topographical deviations as reduced activity on one side could be compensated by enhanced activity on the other side. Evidently, if the active (recording) electrode is in a fixed place for all map configurations, the measured amplitudes optimally reflect the $\mathrm{P} 300$ potential only if the real maximum is located at this position. This type of amplitude assessment is therefore expected to be susceptible to artifact due to topographical asymmetries of the electric field. Such an effect may be crucial for the evaluation of amiplitudes in subject groups with asymmetrical fields such as schizophrenics. The result of no amplitude differences between schizophrenics and controls when using a global estimator of the field strength (reference-independent amplitude) supports this view and leads to the question of whether standard midline amplitudes are an adequate estimator of the P300 field strength (Strik et al 1933b).

Although it is generally accepted that multichannel recordings of the brain's electricial activity are more meaningful for some purposes than recordings with few electrodes, the features of an evoked potential map cannot be easily described and tested as a whole. Accordingly, topographical map differences between groups in most studies are computed as multiple local differences, and the standard estimator for P300 field strength is still the amplitude at prefixed, selected electrode sites (usually $\mathbf{P z}$ ).

Lehmann (1987) has shown that statistical handling of the entire potential maps is possible based on space-oriented data reduction and extraction of meaningful parameters. For a global assessment of amplitudes he has proposed measures that are independent from the recording reference, and includes the potential values of all electrodes.

To clarify the extent and type of interactions between amplitudes and topography of $\mathrm{P} 300$, in the present study we investigated whether (a) asymmetry of the P300 field (i.e., lateralized peak) generally implies low, high, or normal amplitudes, (b) an interaction of the amplitudes with laterization of the peak to one specific side (left or right) can be found, and (c) whether possible interactions differ between standard and reference-independent amplitude measures, and between patients and controls.

The hypothesis was that the P300 electrical fields with lateralization of the peak to either side imply lower amplitudes with the standard assessment at the midline parietal electrode, but not with the reference-independent assessment. No effect of asymmetries to one specific side, and no difference of the extent and type of amplitude-topography interactions between schizophrenic patients and controls were expected.

\section{Methods}

Forty-one schizophrenic inpatients were studied after remission of the acute psychosis [Brief Psychiatric Rating Scale (BPRS) total score: $34.0 \pm 9.66$; Schedule for Assessment of Negative Symptoms (SANS) global score: $10.2 \pm$ 6.5]. All patients met the criteria of schizophrenia according to the DSM-IIl-R (33 residual, 8 remitted). Diagnoses were made independently by two senior psychiatrists and particular attention was paid to the differential diagnosis of mood disorders. Mean age was $33.3 \pm 11.0$ years, mean duration of illness (time since first in- or outpatient treatment) was $9.1 \pm 9.5$ years. Because all the patients were clinically stabilized, medication was low $(277.4 \pm 224.3 \mathrm{mg}$ Chlorpromazine (CPZ)-equivalents). Thirty-nine patients were right-handed, I patient was ambidextrous and I patient was left-handed. None of the patients had concomitant neurological or medical diseases, alcoholism or drug abuse, and none of them had received medication with carbamazepine, lithium, antidepressants, or benzodiazepines.

The control group consisted of 31 right-handed normal controls from the hospital staff and their relatives. Mean age was $29.2 \pm 11.3$ years. No history of neurological or psychiatric disorders or drug abuse was found.

The neurophysiological investigation was based on a standard auditory oddball paradigm with randomly ordered binaural stimuli ( $90 \mathrm{~dB}$ tone burst; duration: $50 \mathrm{msec}$, rise and fall time: $10 \mathrm{msec}$, stimulation rate $0.5 / \mathrm{sec}$ ). A low pitched $(100 \mathrm{~Hz})$ frequent tone had to be ignored, and a rare, high pitched tone $(2000 \mathrm{~Hz}$; presentation rate 1/5) had to be counted by the subject. Online artifact detection supplied by the system (BioLogic Brain Atlas) rejected trials containing eye movements based on an amplitude window. The trial block was continued until 30 artifact-free trials elicited by rare tones were obtained. At the end of the trial block subjects had to report the number of rare tones they had heard. Task execution was considered adequate if the counting error did not exceed $\pm 10 \%$. 
A

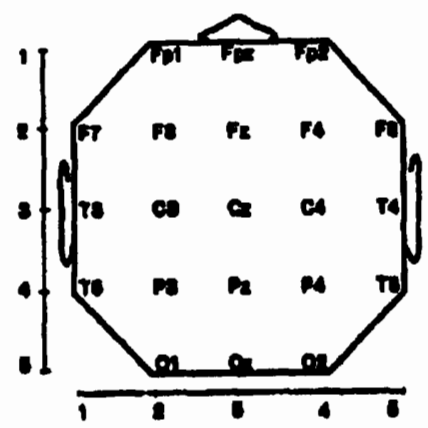

B

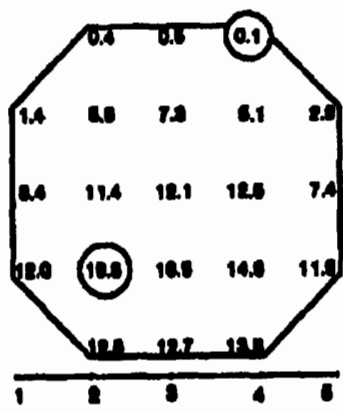

C

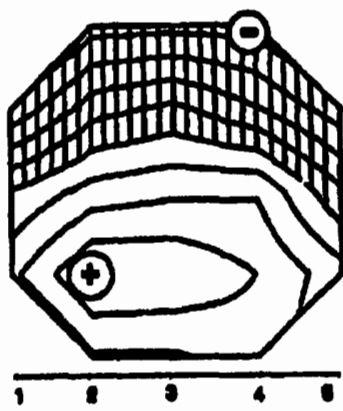

Figure 1. Feasure extraction of multichannel recordings. Top of each panel is anterior, left is left side of the head. (A) Planar projection of the electrode array with 21 electrodes placed according to the international 10-20 system. Fpz was interpolated by the system. The coordinate system displayed at the left and below the graphs allows guantification of each electrode location with one number in the anterior-posterior, and one number in the left-right direction. (B) Example of the measured values in $\mu \mathrm{V}$ at each electrode position at the moment of $\mathrm{P} 300$ in one of our controls. Circles are around the peak $(16.8 \mu \mathrm{V})$ and the trough $(0.1 \mu \mathrm{V})$ of the field. (C) Electrical field map constructed with the values in (B) by interpolation and subtraction of the average reference. The dashed area indicates the negative area of the field after subtraction of the average reference. The location of the peak and the trough (indicated with + and - ) and the relative potential differences (i.e., the equipotential lines) in the field are not affected by this subtraction procedure.

Recordings were obtained from 20 electrodes according to the 10-20 system (see Figure 1A); Fpz was interpolated by the system. Recording reference were linked mastoids with resistors of $10 \mathrm{kOhm}$ each. After filtering (bandpass 0.3-70 $\mathrm{Hz}$ ) and $A D$ conversion (rate: $250 \mathrm{~Hz}$ ) data were stored on disk. Prior to analysis, all files were visually inspected for artifacts.

All P300 parameters were determined in the time window from $270-470 \mathrm{msec}$, and all subsequent statements refer to this window.

As a standard amplitude measure, the peak at the active electrode (Pz), was detected and its amplitude referred to linked mastoids after prestimulus baseline correction (220 msec predelay) was determined.

As reference-independent measures of the P300 field strength we relied on the methods proposed by Lehmann (1987; see also Dierks and Maurer 1990). The parameters utilized were (a) the Global Field Power (GFP), which, in multichannel recordings, is an estimator of the electrical field strength. This one-number parameter is calculated as the "spatial" SD of all measured potential values (excluding the reference) at a given time point (for example, the SD of all values in Figure 1B). (b) The range of the potential values, which we will refer to as the reference-independent amplitude is computed detecting the extreme values (the peak and the trough) of the entire electrical field and calculating the potential difference. In our example in Figure 1B, the highest and lowest value are circled and their difference, that is, the reference independent amplitude at this time point is $16.7 \mu \mathrm{V}$. This parameter is completely independent of the reference, as no reference position will alter the potential difference between two given electrodes. Furthermore, this value provides the advantage of detecting and measuring the effective $\mathrm{P} 300$ peak not only in typical, but also in asymmetrical P300 fields, that is, in fields with lateralized peaks. (c) The location of the $\mathrm{P} 300$ peak was assessed relying on a coordinate system ranging from 1 to 5 in the anterior-posterior and in the left-right direction (see Figure 1). The location of each electrode position is thus identified by two values, for example, the position of the T5-electrode is coded with a 4 in the anterior-posterior direction, and with a 1 in the left-right direction. The code for the lateral position of the peak in Figure IC is 2 . Because field configurations in one time instant usually are simple (one peak, one trough), this procedure provides meaningful data reduction and allows statistical comparisons of topographical configurations independent of absolute voltages.

Because the locations of the extreme values of the map were not normally distributed, a nonparametric statistic was applied for the statistical investigation of interactions between amplitudes and topography. The locations of the P300 peaks between patients and controls were tested in the left-right direction based on their lateral coordinates (see Figure 1; Mann-Whitney U-test). The effect of lateralization of the P300 peak to either side (left or right) on amplitudes was investigated for both groups (patients and controls) testing P300 amplitudes of maps with midline peak against amplitudes of asymmetric maps (left- or right-later- 
Controls

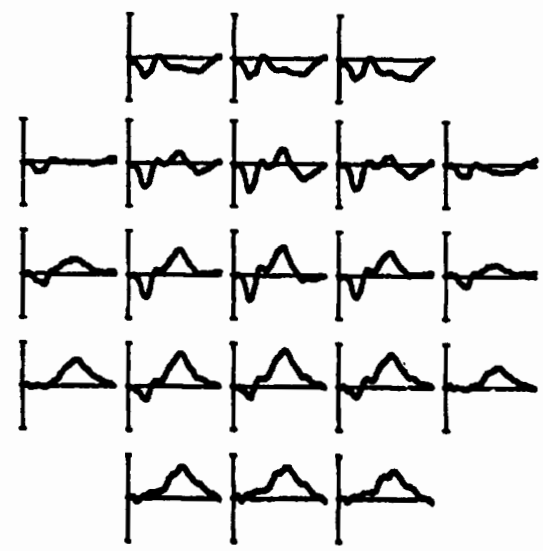

Schkophrenlea

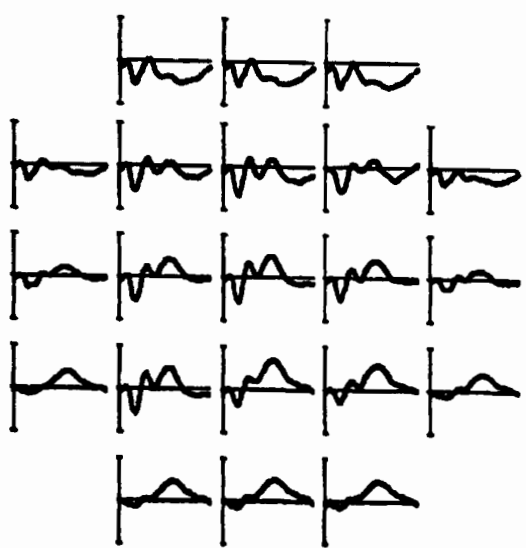

Figure 2. Grand mean of the waveshapes of the evoked potentials elicited by rare tones. All electrode sites are displayed according to the array in Figure 1A. Top of the figure is anterior, left is left side of the head. Waveshapes over left temporal regions (T3 and T5) are smaller in the schizophrenic group.

alized peak; Mann-Whitney U-test); to explore the effect of lateralizations of the P300 peak to one specific side on amplitudes, the locations of the peaks in the leit-right direction were correlated with amplitudes (Spearman's rank correlation); the differences of amplitudes and latencies between patients and controls were tested with $t$-tests. All reported probability levels are two-tailed, with the exception of the standard P300 amplitudes at $\mathrm{Pz}$ between patients and controls, for which confirmatory statistics were computed based on the substantial amount of data from other studies (e.g., Roth and Cannon 1972; Roth et al 1980; Pritchard 1986) which have repeatedly demonstrated reduced amplitudes in schizophrenia.

\section{Results}

The wave shapes of the evoked potentials at all electrode sites (Figure 2) and the P300 electrical field maps (Figure 3) are displayed. Note that the configuration of the equipotential lines of the maps is identical for any reference (including the average reference). The only influence of the reference is on the "sea level", that is, on the relative extensions of the positive and negative (cross-hatched) areas in our map examples. A comparison between different amplitude measures is displayed in Figure $\mathbf{4}$ for patients and controls.

To investigate whether our data replicated the results usually found in schizophrenics, we tested the standard amplitudes at $\mathrm{Pz}$ and the left-right locations of the P300 peaks for group differences. The standard P300 amplitudes at the $\mathrm{Pz}$ electrode were lower in schizophrenics than in controls (controls: $10.4 \pm 4.1 \mu \mathrm{V}$, schizophrenics: $8.1 \pm$
$6.3 \mu \mathrm{V}, t=1.87, p<0.05$ ). The peak of the $\mathrm{P} 300$ field was located significantly more on the right in schizophrenic patients with respect to controls who had slightly left-sided peaks with respect to midline $(3.27 \pm 0.95$ versus $2.55 \pm$ $0.81 ; z=2.93, p=0.003$; for illustration of the coordinate values see Figure $1 \mathrm{~A}-\mathrm{C}$ ). This difference appears in the form of slightly lower grand average amplitudes in left temporal regions (Figure 2), but can hardly be seen in the grand average maps (Figure 3 ).

P300-latencies and the reference-independent amplitudes were tested for a comprehensive layout of the data. No differences were found between patients and controls (latencies: controls $349.4 \pm 43.1 \mathrm{msec}$, schizophrenics $363.3 \pm 49.6 \mathrm{msec}, t=1.25$, ns; reference-independent amplitudes: controls: $16.5 \pm 5.3$, schizophrenics $16.0 \pm$ 9.7. $t=0.27$; ns).

Asymmetric topography, that is, left or right lateralized P300-peak, was associated with lower standard ampli!.des (amplitude at the Pz-electrode) compared to symmetric P300 fields with peak at midline. This was true for the entire group, including patients and controls. No such difference was found for the reference-independent amplitude (Table 1).

Unexpectedly, in schizophrenics, the standard amplitudes correlated significantly and inversely with the leftright coordinates of the locations of the maximal positivity. In other words, the farther the P300 peak was at the right side, the lower was the amplitude at Pz (Spearman's rank correlation: $p=-0.63, p<0.00001)$. This correlation was also found for the reference-independent amplitude $(\rho=$ $-0.39, p=0.012$ ). No correlations were found in controls 

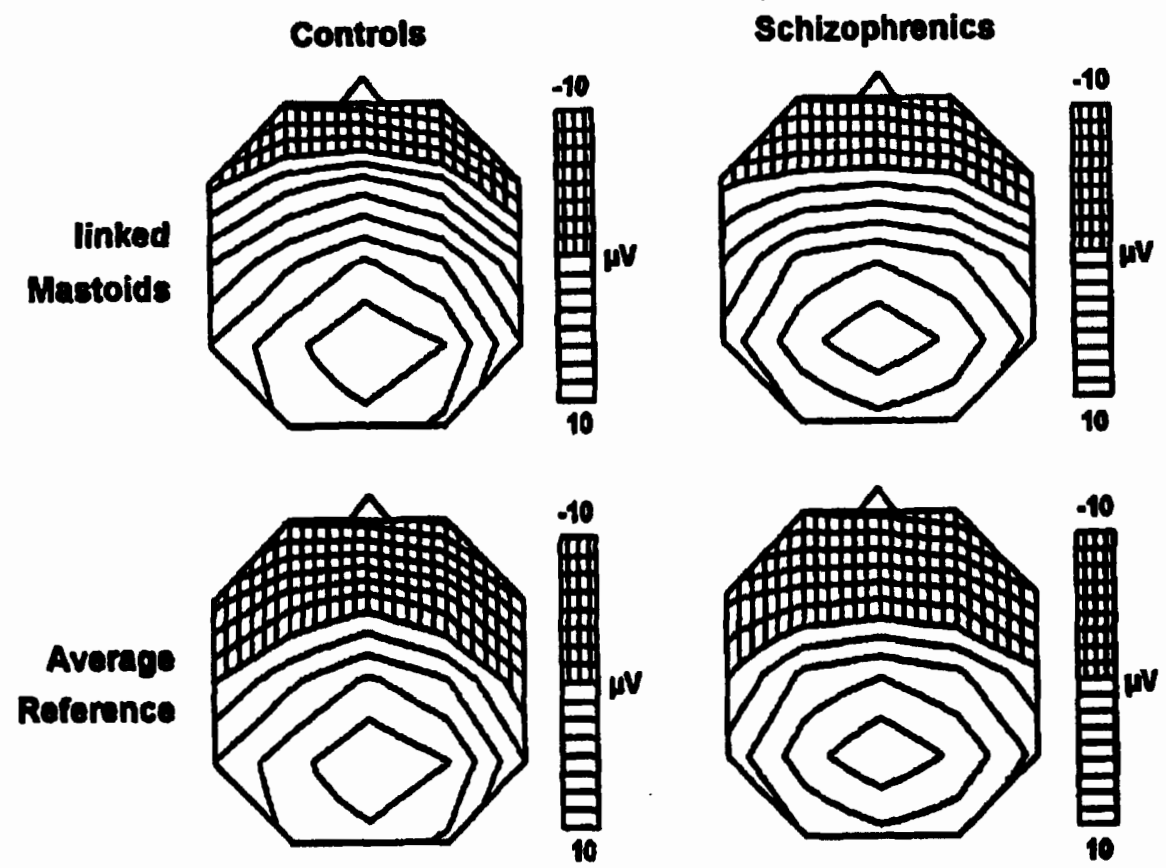

Figure 3. Grand average maps of the P300 fields at the latency of the grand averages. Comparison between maps referted to linked mastoids (top) and to average reference (bottom); view from top. The topographical configurations of the maps do not differ for the different references, only the relative extension of the negative (cross-hatched) and the positive area are affected. The topographical differences between schizophrenics and controls are blurred in the grand mean maps due to the predominance of maps with midline peak and high amplitudes (see Figure 5). This difference can hardly be seen in the grand average maps.

(standard: $p=0.18$, ns; reference-independent: $p=0.26$, ns; Figure 5).

\section{Discussion}

The extent and type of interactions between topography and amplitudes in normals and in schizophrenics, although the subject of speculation, have not beel documented previously. Such an interaction is relevant, because even in multichannel studies only P300 amplitudes at midline are reported as estimators of the field strength, and P300 asymmetries in schizophrenics have been repeatedly demonstrated (Morstyn et al 1983; Faux et al 1990; Strik et al 1993a). In the present study, we found the standard P300 amplitude at midline electrodes to be significantly asso. ciated with topographical asymmetries of the electrical field. This effect was found in patients and in controls, and was present for left-lateralized and for right-lateralized P300 peaks. The reference-independent amplitude as a global estimator of the P300 field strength, on the other hand, appeared to be robust to this interaction. In addition, in the schizophrenic group, there was a significant effect of the direction of asymmetry, with lower amplitudes associated with right-lateralized $\mathrm{P} 300$ peaks. This was true for standard and for reference-independent amplitudes. No similar effect of direction was found in the control group. Furthermore, the major findings in the literature of lower standard amplitudes in schizophrenics, of left-lateralized P300 peaks in controls, and of right-laterelized peaks in schizophrenics were replicated.

The patients investigated in this study were in a stabilized, remitted, or residual state. This may explain the weak significance of the difference between patients and controls in the conventional amplitude assessment at the Pz-electrode, as P300 amplitude tends to increase after successful treatment (Duncan et al 1987) and patients with cycloid psychoses that fulfill DSM-III-R criteria for remitted schizophrenia have normal amplitudes (Strik et al 1933a). The lack of group difference of the reference-independent amplitudes is in line with the previous findings of our group that led to the hypotheses of the present study (Strik et al 1993a).

Considering the P300 field as the sum of widespread neuronal activity, the observed lower standard amplitudes in case of asymmetric topography were expected as arifacts of the standard amplitude assessment at prefixed electrode sites. The reference-independent amplitude as an estimator of the P300 field strength, on the other hand, was not lower 

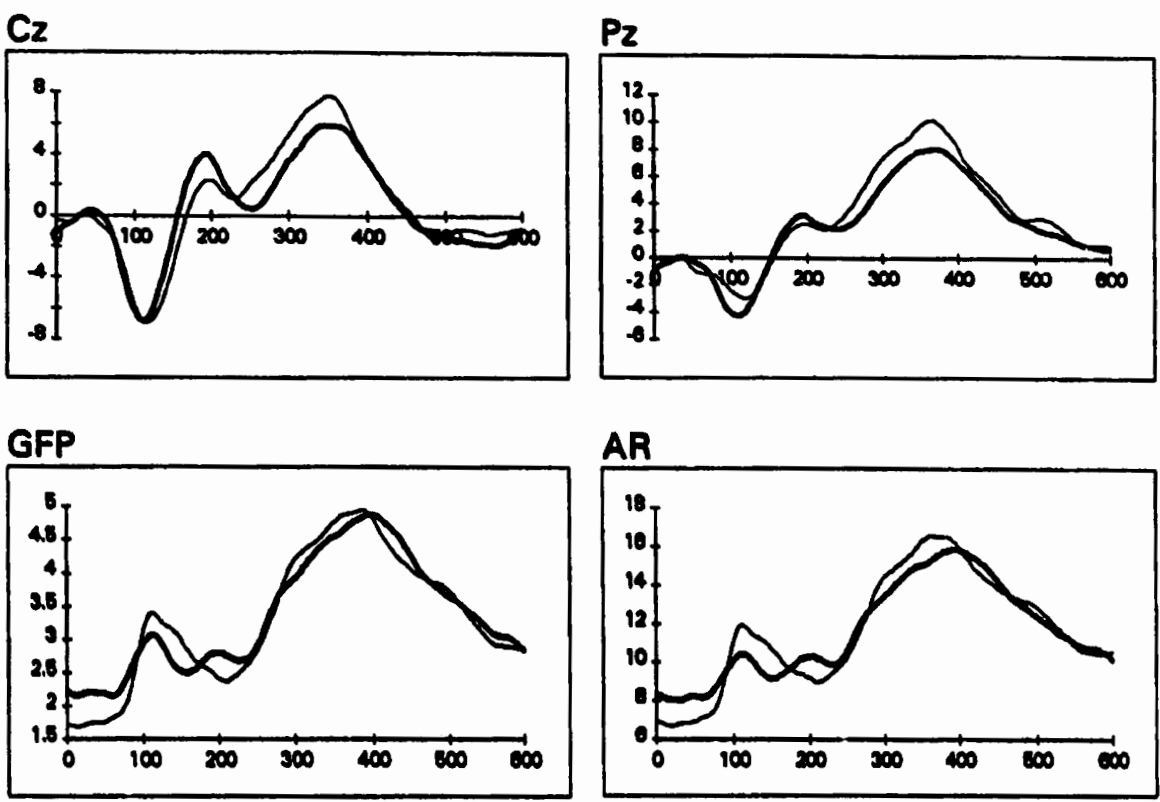

Figure 4. Evoked potential curves of different estimators of the strength of the P300 field. Bold line: schizophrenic patients; thin line: controls. Units are milliseconds ( $x$ axis) and $\mu$ Volts ( $y$ axis). In the upper part, ERP waveshapes at the central $(\mathbf{C z})$ and midline parietal $\left(\mathrm{Pz}_{2}\right)$ electrodes are displayed. Waveshapes were obtained after prestimulus baseline subtraction $(220 \mathrm{msec}$ predelay); Below, the curves of the Global Field Power (GFP) and of the reference independent amplitude (amplitude range, AR).

even if the $\mathrm{P} 300$ peak was lateralized. This indicates that asymmetry per se is not necessarily a sign of reduced strength of the underlying generators.

Contrary to the preceding finding, the significant amplitude-reducing effect of right-sided $\mathrm{P} 300$ peaks in the schizophrenic group was unexpected. This does not appear to be a methodological artifact, as it was also found in referenceindependent amplitudes. Because normal P300 often is asymmetric with a left-sided peak (Morstyn et al 1983, Strik et al 1993a; see also Figure 5), one might expect an inverse

Table 1. Amplitudes of P300 Fields with Symmetric (Midline Peak) and Asymmetric (Lateralized Peak) Configuration

\begin{tabular}{lcccc}
\hline & Symmetric & Asymmetric & $|z|$ & $p$ \\
\hline Schizophrenics & & & & \\
$n$ & 20 & 21 & & \\
Pz & $13.1 \pm 5.7$ & $8.7 \pm 5.0$ & 2.39 & 0.017 \\
Ampl. range & $21.5 \pm 9.5$ & $17.7 \pm 8.4$ & 1.38 & NS \\
Controls & & & & \\
$\quad n$ & 14 & 17 & & \\
Pz & $14.7 \pm 4.8$ & $11.2 \pm 3.8$ & 2.06 & 0.039 \\
Apl. range & $20.1 \pm 5.3$ & $18.6 \pm 5.6$ & 0.99 & NS \\
\hline
\end{tabular}

Means and SDs of amplitudes with symmetric (P300 peak over midline) and asymmetric (left-lateralized or right-laternlized peak) P300 field configurations. Standard amplitudes were lower if the peak was lateralized. No significan differences werefound for reference-independent amplitudes. Tests were compured with MannWhitney's $U$-test, probability levels are two-tailed. correlation in the control group, but no such effect was found. This finding is relevant for the interpretation of the right-lateralized P300 peaks in schizophrenic patients.

Lower amplitudes when the P300 peak is over the right hemisphere, no effect or an inverse effect for left-sided asymmetries in the schizophrenic group (see Figure 5), and no effect of any specific side in the control group suggests that the deviations to the right of the $\mathrm{P} 300$ peak in schizophrenic patients are due to a functional deficit of left hemispheric generators. This interpretation is supported by the recent findings of McCarley et al (1993; see Introduction). Contrarily, asymmetries with left-sided peaks in both, schizophrenics and normal controls, seem to be due to a physiological left hemispheric predominance during the cognitive task rather than to right-hemispheric functional deficits, because they are not accompanied by amplitude reductions.

As can be easily seen in Figure 5, many of our stabilized schizophrenic patients have normal locations and amplitudes of the $P 300$ peaks. The result suggests the subdivision of schizophrenic patients into those with central- or leftsided P300 peak and normal amplitudes, and those with low P300 amplitudes and right-hemispheric deviations. After remission of the acute psychosis, these latter features may be related to an insidious long-term course and to pervasive cognitive impairment of a subgroup of schizophrenic patients (Strik et al 1993b). 
Controls

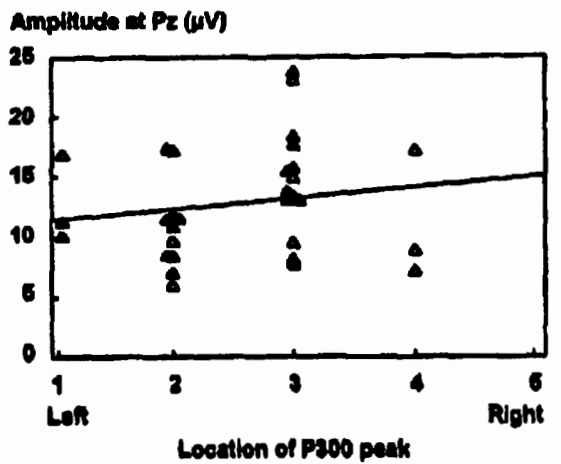

Schizophrenics

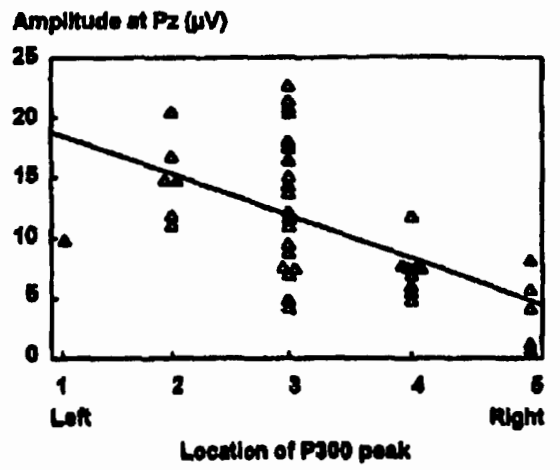

Figure 5. Scatter plot of P300 amplitudes (y axis; amplitudes are measured at the individual peaks at $P_{2}$ ) versus location of the $\mathbf{P 3 0 0}$ maximum ( $x$ axis). Locations are coded in the left-right direction with one integer value from I to 5 (see Figure 1). In the schizophrenic group there was a significant correlation between right hemispheric deviations of the maximum and low amplitudes. No correlations were found in the control group.

In conclusion, there are relevant interactions between P300 amplitudes and asymmetrical field configurations. The overall field-strength, as expressed by the referenceindependent amplitude, is robust to reduction due to asymmetries as a methodological artifact. We suggest to apply this parameter for a more reliable estimation of the field strength in multichannel recordings to avoid confounding of topographical asymmetries with reduced field strength. This is important for group comparisons as well as for the

\section{References}

Dierks T. Maurer K (1990): Reference-free evaluation of auditory evoked potentials - P300 in aging and dementia. In Dostert P, Riederer P, Strollin Benedett M. Roncucel R (eds), Early Markers in Parkinson's and Alzheimer's disease. Berlin: Springer, pp 197-208.

Duncan CC. Morihisa JM, Fawcet RW, Kirch DO (1987): P300 in schizophrenia: State or trait marker? Psychopharmacol Bull 23:497-501.

Faux SF, Shenton ME, McCarley RW, Nestor PG, Marcy B, Ludwig A (1990): Preservation of P300 event-related potential topographic asymmetries in schizophrenia with use of either linked-ear or nose reference sites. Electroencephalogr Clin Neunophysiol 75:378-391.

Halgren E, Stapleton JM. Smith M, Altafullah I (1986); Generators of the human scalp P3(s). In Cracco Q. Bodis-Wollner I (eds) Evoked Potentials. New York: Alan Liss, pp 269-284.

Lehmann D (1987): Principles of spatial analysis. In Gevins AS, Remond A (eds), Methods of Analvsis of Brain Electrical and Magnesic Signals. Handbook of Electroencephalognaphy and Clinical Neurophysiology (rev Vol. I). Amsterdam: Elsevier, pp 309-354.

McCarley RW, Faux SF, Shenton ME, Nestor PG, Holinger DP (1991): Is there P300 asymmetry in schizophrenia? Arch Gen Psychiatry 48:380-381.

McCarley RW, Shenton ME, O'Donnel BF, et al (1993): Auditory investigation of the meaning of the P300 amplitudes in schizophrenia. Discounting this source of artifact, there remains in schizophrenia a specific association of low amplitudes and right-hemispheric deviations of the maximal P300-positivity. This finding can be interpreted as another piece of evidence for asymmetric (probably left hemispheric) functional deficits involving the generators of $\mathrm{P} 300$ in some schizophrenic patients.

P300 abnormalities and left posterior superior temporal gyrus volume reduction in schizophrenia. Arch Gen Psychiatry 50:190-197.

Morstyn R, Duffy FH, McCarley RW (1983): Altered P300 Topography in schizophrenia. Arch Gen Psychiatry 40:729-734.

Pritchard. W.S. (1986): Cognitive event-related potentials correlates of schizophrenia. Psychol Bull 100:43-66.

Roth WT, Cannon EH (1972): Some features of the auditory evoked response in schizophrenics. Arch Gen Psychiarry 27:466-471.

Roth WT, Horvath TB. Pfefferbaum A, Kopell, BS. (1980) Eventrelated potentials in schizophrenics. Electroencephalogr Clin Neurophysiol 48:127-139.

Smith ME, Halgren E, Sokolik M (1990): The intracranial topography of P3 event-related potential elicited during auditory oddball, Electroencephalogr Clin Neurophysiol 76:235-248.

Strik WK, Dierks T, Franzek E. Maurer K, Beckmann H (1993a): Differences in P300-Amplitudes and Topography between Cycloid Psychosis and Schizophrenia in Leonard's Classification. Acta Psychiair Scand 87:179-183.

Strik WK, Dierks T, Maurer K (1993b): Amplitudes of auditory P300 in remitled and residual schizophrenics: correlations with clinical features. Neuropsychobiology 27:54-60.

Wood CC, McCarthy G (1985): A possible frontal lobe contribution to scalp P300. Neurosci Abstr 11:879-884. 